\title{
Research on the Design of Digital Library Cloud Service Platform Based on the Integration of Scattered Digital Information Resources in the Age of Cloud Computing
}

\author{
Yun Fan \\ Yunnan Jiaotong College, Yunnan, Kunming, 650500
}

Keywords: cloud computing; resources integration; digital library; cloud platform

\begin{abstract}
Under the cloud computing era, the digital library cloud service platform is an inevitable trend of digital library development, which can effectively improve the digital library digital information resource decentralization and realize the effective integration and sharing of digital library information resources. Therefore, the paper uses the cloud computing technology to design a digital library cloud service platform from three aspects including platform overall structure, work flow and operation mechanism.
\end{abstract}

Following the personal computer, internet, IT industry has entered into a new period of change, namely cloud computing. The emergence of cloud computing is mainly due to the development of high-speed Internet, virtual technology, data center. It can be said that cloud computing is the product of the current information age. Under cloud computing context, network users obtain what they need through network expansion. The "cloud" resources are unlimited, scalable, and available to users at any time. While this resource is generally not free access, you need to pay a fee based on resource conditions. Through loud computing the centralized storage of data is realized. Besides, users can quickly obtain the required data and information, the way of data leakage is effectively reduced, and the data security monitoring can also be easily implemented. Therefore, cloud computing has many features including computing power, virtualization, reliability, scalability, and reasonable price. Based on these advantages, cloud computing has become the currently widely used Internet technology. Amazon, Google, Microsoft, and other companies all have begun the use of cloud computing.

Under the information age, libraries have also undergone changes and started to develop in the direction of informationization and networking. With the advent of the cloud computing era, libraries have also begun to introduce cloud computing technology, which has led to the creation of digital libraries. In the construction of digital library, how to use the cloud computing to construct a cloud service platform is an inevitable trend of the current digital library development. Therefore, the paper designs a digital library cloud service platform based on the integration of distributed digital information resources in the age of cloud computing.

\section{The overview of cloud service platform in digital library}

Digital library emerges in order to adapt to the development of the library. The construction of the digital realizes the digital management of the library information. And the library service of user personification has been satisfied as well as the digital book reading. In addition, the books information of the digital library is collected dynamically instead of randomly. Then it can be represented to the user. When users search for book information in the digital library, the seamless connection based on digital library greatly facilitates the search and application of the book information of user. These are the advantages of digital library.

But the current construction process of the digital library also exist some shortcomings among which the largest one is the scatter of digital information. The effective information resources have not been integrated and the effect of the digital library information has not been achieved, which may not only lead to the human and financial waste during the management process of the digital 
library, but also affect the construction of digital library. Therefore, based on this situation, in the era of cloud computing, it is imminent to build a digital library cloud service platform to improve the integration and dispersion of digital information resources.

\section{The design of cloud service platform in digital library}

The design of digital library cloud service platform mainly applies cloud computing technology.

Based on the principle of economy and practicability, it realizes the integration of information resources of digital library, and provides convenience for library management and user reading. The following design is carried out from the overall architecture of the digital library cloud service platform.

In the digital library cloud service platform, it mainly includes four layers of application layer, management platform, data layer and infrastructure layer, as shown in Figure 1.

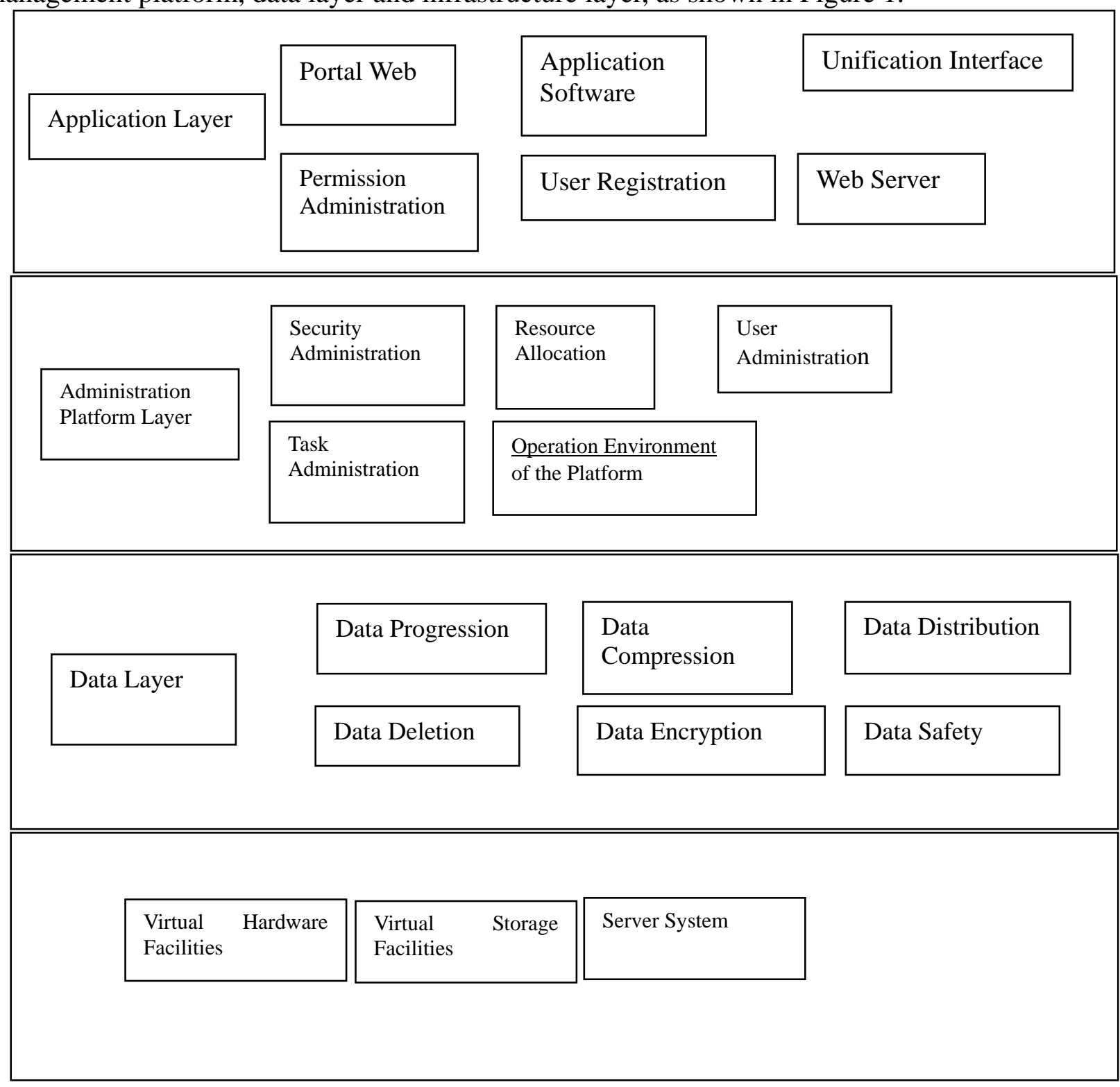

Figure 1 System Structure

Application level: This level mainly includes web portals, application software, unified AIP interface, authority management, user registration, Web Server and so on. Through these, we can make user registration, login function, and various functional modules of the cloud service platform be presented in front of the users, so as to meet the needs of user applications.

Management Platform level: The main achievement of the level is the achievement of security 
management, resource allocation, user management, task management and platform operating environment, it's the foundation of the digital library cloud service platform management activities and so belongs to the core level.

Data level: This level mainly includes data scheduling, data compression, data distribution, data deletion, data encryption, data security and so on, it realizes the data information management of digital library cloud service platform. When designing this, database virtualization technology was adopted and database management was realized through the middleware.

Infrastructure level: This level mainly includes virtual hardware facilities, virtual storage facilities, server systems, server clusters, network resource interconnection, etc., which guarantees the infrastructure for the digital library cloud service platform. When designing this, virtualization technology and embedded cloud terminal technology were adopted to achieve a variety of infrastructure and network connectivity.

\section{The design of digital library cloud service platform}

Digital library cloud service platform works in accordance with the established process to provide users with services. Figure 2 is the workflow diagram for digital library cloud service platform.

\begin{tabular}{|c|c|c|c|c|c|}
\hline $\begin{array}{l}\text { Description of } \\
\text { information } \\
\text { resources }\end{array}$ & $\Longrightarrow$ & $\begin{array}{l}\text { Matching of } \\
\text { information } \\
\text { resources }\end{array}$ & $\Longrightarrow$ & $\begin{array}{l}\text { Allocation of } \\
\text { information } \\
\text { resources }\end{array}$ & $\begin{array}{l}\text { Release of } \\
\text { information } \\
\text { resources }\end{array}$ \\
\hline
\end{tabular}

Figure 2 The workflow of Digital Library Cloud Service Platform

Description of information resources: When a user logs in to the digital library cloud service platform, he or she will issue various types of access requests to the platform according to his needs in the platform interface. For the user's access request, the platform will provide users services based on the Web, and make description for the information resources users need according to user requests. It can be seen that the information resource description is based on the user's various types of access requests, and only after receiving the user request, the platform will describe the information resources, which is the first step in the working mechanism of digital library cloud service platform.

Information resource matching: Information resource matching is the second step in the working mechanism of digital library cloud service platform, which follows the description of information resources, the foundation of information resource matching. After the information resource is described according to the user's access request, data search, data collection and information resource matching requested by users are conducted in database according to the described information. The effect of information resources matching would be influenced by infrastructure of the platform, which should be improved and optimized to ensure the high-efficiency and integrity of information resource matching.

Information resource scheduling: As the third step in the working mechanism of digital library cloud service platform, Information resource scheduling mainly implements the effective scheduling of all kinds of information resources. The stored information resources are planned and unified on the digital library cloud service platform. In order to ensure the consistency of information resources and access requests, information resources need to be scheduled to understand whether the information resources stored in the database can meet the user's access requests and thus provide users with accurate and complete information resources.

Release of information resources: Information resources release, the last step in the working mechanism of digital library cloud service platform, is to release the found information resources for users to use. The release of information resources is the result of the above three steps, which is also a manifestation of realizing the user access request. 


\section{Design of operation mechanism of digital library cloud service platform}

The digital library cloud service platform is a complex and tedious system, whose operation mechanism needs to be designed in order to ensure the stability and security of the operation process. Figure 3 shows the flow chart of digital library cloud service platform operating mechanism.

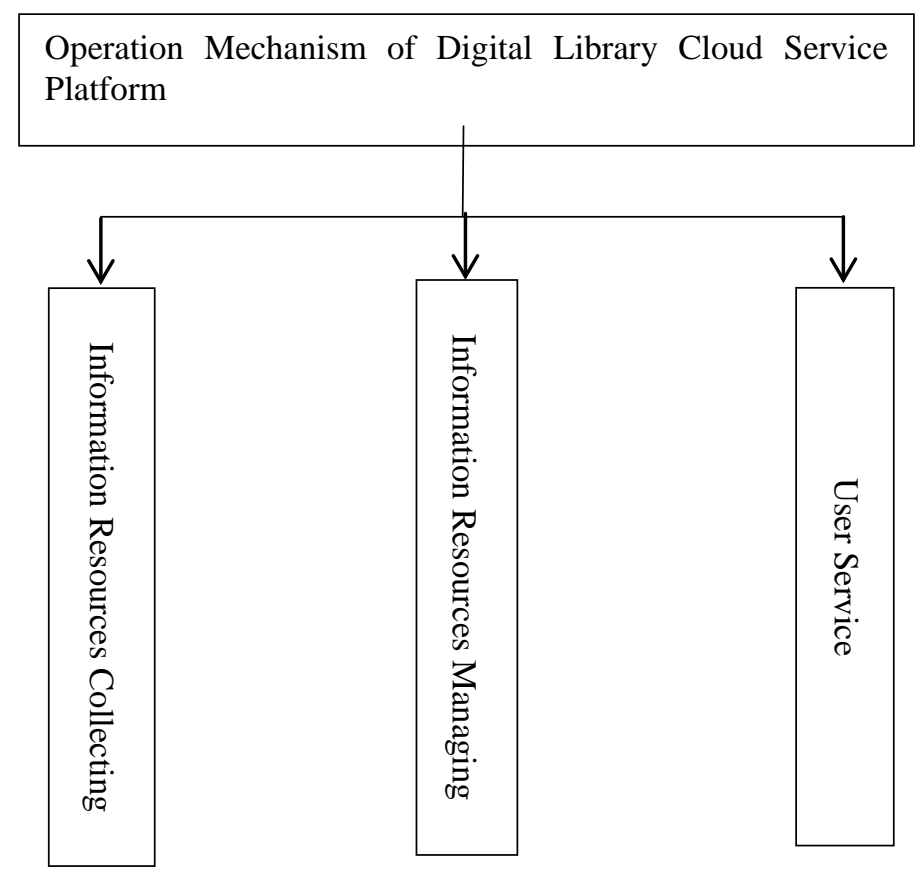

Figure 3 Operating mechanism flow chart

In the operation mechanism of digital library cloud service platform, it mainly includes three mechanisms of information resource collection, information resource management and user service.

Information resource collection: Information resource collection is the basis of meeting the user's request. After the user makes a request in the platform, the platform collects the qualified information resources in the cloud platform database according to the request, and screens the information resources through information matching and information scheduling to meet the user's request.

Information resource management: digital library cloud service platform has stored a large number of digital books information, so the information resource management is needed. The information resource management has been done mainly through the application software. The use of virtual technology for all types of information resources will be that normative, reasonable management, to achieve the effectiveness of various types of information resources, and lay a good foundation for the utilization of information resources in the later period.

User Service: Providing digital book information to users is the purpose of constructing the digital library cloud service platform. In order to fully meet the needs of users, a user service mechanism is required. After the user making a request in the platform, the platform analyzes firstly, then processes and matches the request, then publishes the information that meets the user's request, and accurately as well as quickly reflects the user's request.

Cloud computing is the mainstream information technology in the coming period and will exert a tremendous influence and effect in various industries. Digital library should follow the trend of the times, in view of the current situation of decentralized information resources. Its cloud computing technology carries out research on cloud service platform to improve the integration and sharing of information resources to achieve the role of digital libraries.

\section{References}

[1] Zhang Junling. Research on the Integration Mechanism of Information Resources of University 
Digital Library in the Cloud Computing Environment[J]. Library Science Research, 2012(7):25-28.

[2] Yu Xin, Research on the Cloud Services Platform Architecture of Digital Library Based on Cloud Computing Technology[J]. Information Science, 2011(7):1049-1053.

[3] Chen Gong, Niu Qinzhou. Digital Library Information Service Platform Based on Cloud Computing[J]. Information Science, 2012(5):684-688. 